\title{
Clinicopathological association and prognostic value of long non-coding RNA CASC9 in patients with cancer: A meta-analysis
}

\author{
HOU-YU DUAN, XIANG DING and HE-SHENG LUO
}

\author{
Department of Gastroenterology, Renmin Hospital of Wuhan University, Wuhan, Hubei 430060, P.R. China
}

Received April 11, 2020; Accepted July 1, 2020

DOI: 10.3892 /etm.2020.9096

\begin{abstract}
Several studies have reported a prognostic role of the long non-coding RNA (lncRNA) cancer susceptibility candidate 9 (CASC9) in various cancer types, but its clinical significance has remained inconclusive. The aim of the present meta-analysis was to evaluate the impact of CASC9 expression on the prognosis and clinicopathological features of patients with cancer patients. The PubMed, Embase, Cochrane Library and Web of Science databases were searched for relevant literature and eight studies, including 565 patients with cancer, were selected. The quality of these studies was appraised with the Newcastle-Ottawa Scale (NOS) and the association between CASC9 expression and prognosis or clinicopathological features was analyzed. Patients with high expression levels of CASC9 in their tumor tissues had a lower overall survival rate compared with those in the low CASC9 expression group (hazard ratio $=2.25,95 \% \mathrm{CI}: 1.60-3.17, \mathrm{P}<0.001$ ). Furthermore, elevated CASC9 expression was significantly associated with deeper tumor invasion [odds ratio $(\mathrm{OR})=2.66,95 \% \mathrm{CI}$ : 1.72-4.14, $\mathrm{P}<0.001]$, poor tumor differentiation $(\mathrm{OR}=2.44$, 95\% CI: 1.24-4.78, $\mathrm{P}=0.009)$, lymph node metastasis $(\mathrm{OR}=3.42,95 \% \mathrm{CI}: 1.98-5.92, \mathrm{P}<0.001)$ and advanced clinical stage $(\mathrm{OR}=3.21,95 \% \mathrm{CI}: 2.21-4.66, \mathrm{P}<0.001)$. In conclusion, CASC9 is a promising biomarker for predicting the prognosis of cancer patients and should be validated in the clinic.
\end{abstract}

\section{Introduction}

Cancer is one of the leading causes of death worldwide (1). The outcomes of the current therapeutic modalities, including

Correspondence to: Professor He-Sheng Luo, Department of Gastroenterology, Renmin Hospital of Wuhan University, 238 Jiefang Road, Wuhan, Hubei 430060, P.R. China

E-mail: xhnk@163.com

Abbreviations: CASC9, cancer susceptibility candidate 9; DFS, disease-free survival; HR, hazard ratio; lncRNA, long non-coding RNA; NOS, Newcastle-Ottawa Scale; OR, odds ratio; OS, overall survival; PFS, progression-free survival

Key words: long non-coding RNA, CASC9, prognosis, cancer, meta-analysis surgery, radiotherapy, chemotherapy, biotherapy and palliative treatments, are far from satisfactory. Therefore, it is essential to identify novel markers with high sensitivity and specificity in order to improve the early diagnosis and prognosis of patients with cancer. Long non-coding RNAs (lncRNAs) have gained considerable attention as reliable cancer biomarkers. LncRNAs are transcripts longer than 200 nucleotides that lack an open reading frame and protein-coding capacity $(2,3)$. They constitute $>80 \%$ of the entire transcriptome and have long been considered as transcriptional 'noise' $(4,5)$. However, more recent studies have indicated that lncRNAs have significant roles in cancer progression $(6,7)$. The gene encoding the lncRNA cancer susceptibility candidate 9 (CASC9) is located on the human chromosome $8 \mathrm{q} 21.11$ and has four isoforms (CASC9-001 to -004), of which CASC9-004 is commonly referred to as CASC9 due to its relatively high prevalence $(8,9)$. CASC9 was initially identified in esophageal squamous cell carcinoma (ESCC) (10) and has since then been detected at aberrantly high levels in nasopharyngeal carcinoma (11), oral squamous cell carcinoma (OSCC) (12), lung squamous cell carcinoma (13), breast cancer (BC) (14), gastric cancer (GC) (15), hepatocellular carcinoma (9), pancreatic ductal adenocarcinoma (16), colorectal cancer (CRC) (17), ovarian cancer (OC) (18) and cervical cancer (19). Furthermore, high expression of CASC9 is associated with poor prognosis and unfavorable clinicopathological parameters in cancer patients $(12,15,17,18,20-23)$. Since individual studies are limited by relatively small sample sizes and discrete outcomes, a systematic analysis is necessary in order to obtain more insight into the role of CASC9 in human cancers. To this end, a meta-analysis was performed in the present study to evaluate the prognostic and clinicopathological significance of CASC9 in cancer.

\section{Materials and methods}

Literature search strategy. Relevant articles published in the English language till November 30, 2019 were retrieved from the PubMed, Embase, Cochrane Library and Web of Science databases by two independent researchers (HYD and XD). According to the Cochrane systematic evaluation manual 5.1, the following MeSH terms and free-text words were combined for the literature search: 'long noncoding RNA CASC9' OR 'IncRNA CASC9' OR 'long non-coding RNA CASC9' OR 'cancer susceptibility 9 lncRNA' OR 'cancer susceptibility 9 long noncoding RNA' OR 'CASC9 non-coding long RNA' and 'Neoplasia' OR 'Neoplasias' 


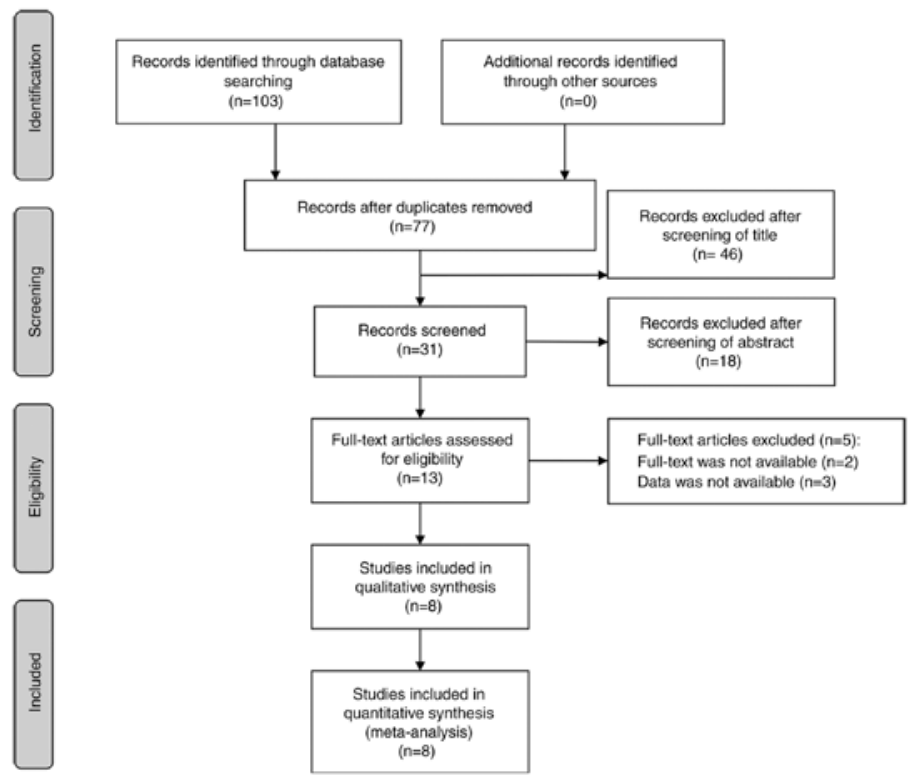

Figure 1. Flowchart of the study selection procedure.

OR 'Neoplasm' OR 'Neoplasms' OR 'Tumors' OR ‘Tumor' OR 'Cancer' OR 'Cancers' OR 'Malignancy' OR 'Malignancies'. The bibliography of the selected studies was manually examined to identify any further relevant studies. In the case of insufficient data, the authors were contacted to request the relevant information via e-mail. The articles were preliminarily screened on the basis of their titles and abstracts, and their eligibility for the meta-analysis was determined by two researchers after full-text analysis. The meta-analysis was performed according to the Preferred Reporting Items for Systematic Reviews and Meta-Analyses guidelines (24). Any disagreements were resolved by a third researcher (HSL).

Literature selection. The inclusion criteria for the studies were as follows: i) Published clinical research, ii) published in the English language, iii) including patients with histo-pathologically confirmed cancer, iv) reporting on the association between CASC9 expression and clinicopathological features or prognosis of cancer patients. Studies that did not fulfill the inclusion criteria, literature reviews, editorials, letters and case reports, as well as studies lacking full text or relevant data, were excluded.

Data extraction. Basic information on the studies, including the first author's name, year of publication, country, sample size, tumor type, method of detection, cut-off value, survival analysis type and Newcastle-Ottawa Scale (NOS) scores, as well as clinicopathological features of the patients, including sex, age, tumor size, depth of invasion, tumor differentiation, lymph node metastasis and clinical stage, and outcome indicators such as overall survival (OS), disease-free survival (DFS) and progression-free survival (PFS), were extracted. Certain outcome indicators were directly acquired from the studies and others were extracted from Kaplan-Meier survival curves using hazard ratio (HR) digitizer software Engauge Digitizer 4.1 (25).

Quality assessment. The NOS (26) was used for evaluating the quality of included studies. This quality assessment method evaluated studies by eight items, which were categorized into three aspects: Patient selection (4 items), study comparability (1 item) and study outcome (3 items) (27). An answer of 'yes' to each question of the items was counted as the score of 1 ; otherwise, a score of 0 was awarded (26). The NOS scores ranged from 0 to 9 and studies with scores of $\geq 6$ were considered to be of high quality. Any disagreement concerning the quality assessment of studies was resolved by discussion until consensus was reached among the researchers.

Statistical analyses. Review Manager 5.2 (The Cochrane Institute) and Stata 12.0 software (StataCorp.) were used for statistical analysis. Since the meta-analysis was performed on previously published studies, no ethical approval was required. The association between CASC9 expression and OS rates was evaluated by determining the $\mathrm{HR}$ and $95 \% \mathrm{CI}$, and that between CASC9 expression and clinicopathological features by determining odds ratios (ORs) and $95 \%$ CIs. A $\chi^{2}$-based Q-test and $\mathrm{I}^{2}$ statistics were used to estimate the heterogeneity of the included studies. A fixed-effects model was used in case of insignificant heterogeneity $\left(\mathrm{I}^{2}<50 \%\right)$, and otherwise, the random-effects model was used for meta-analysis. Sensitivity analysis was performed using Stata 12.0 software to determine the robustness of the meta-analysis results. The potential publication bias was assessed using Begg's funnel plot and Egger's test (28). All P-values were two-tailed and $\mathrm{P}<0.05$ was considered to indicate statistical significance.

\section{Results}

Characteristics and quality of included studies. Based on the search strategy, 103 papers were initially retrieved, of which 13 full-text articles were evaluated for their eligibility after preliminary screening of titles and abstracts. Finally, eight studies in including 565 patients were selected $(12,15,17,18,20-23)$. The screening procedure is outlined in Fig. 1. The included studies were published between 2016 and 2019, and the most 

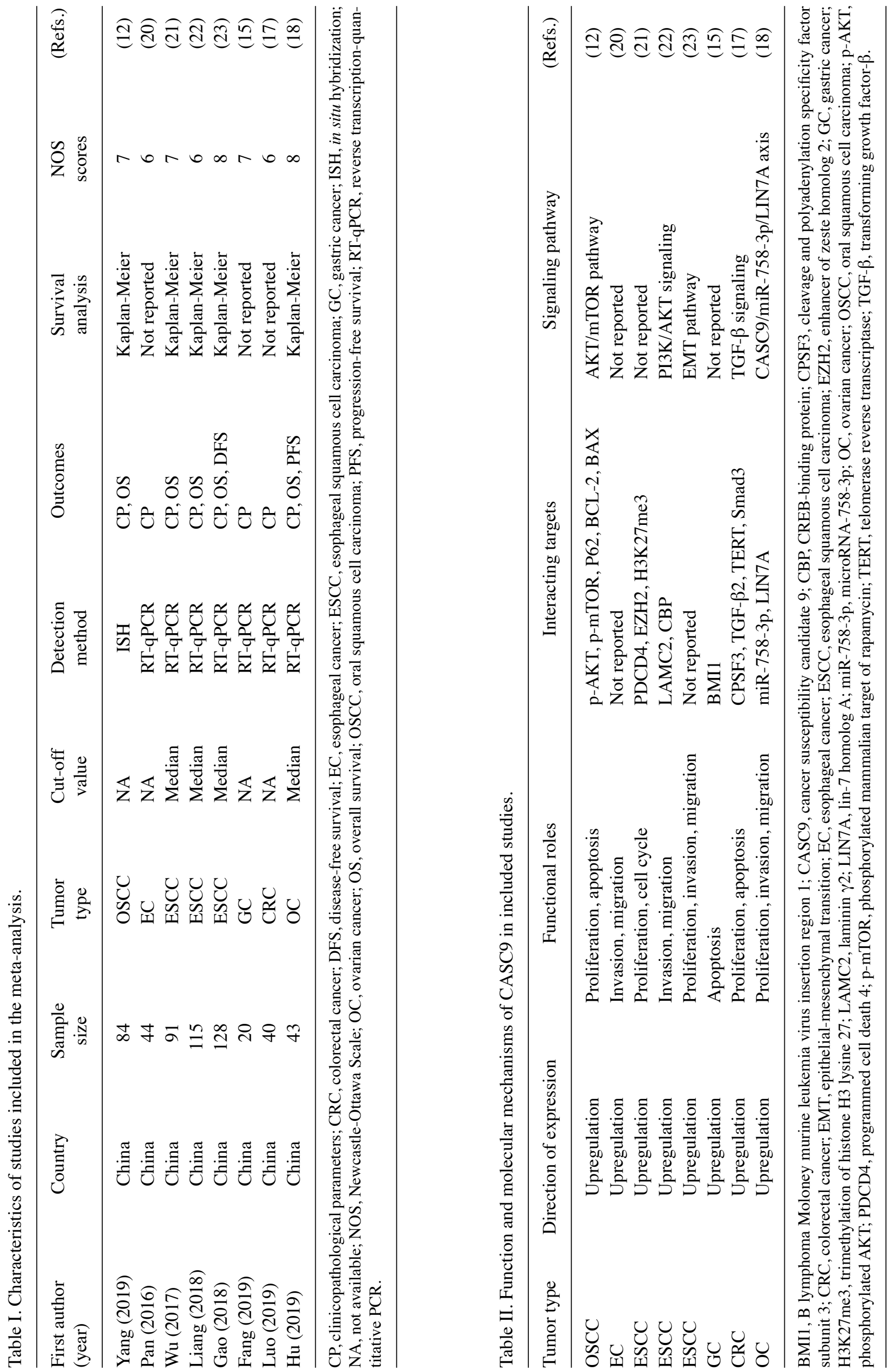
Table III. Quality assessment of included studies using the Newcastle-Ottawa Scale.

\begin{tabular}{lccccc}
\hline First author (year) & Selection & Comparability & Outcome & Total scores & (Refs.) \\
\hline Yang (2019) & 4 & 1 & 2 & 7 & $(12)$ \\
Pan (2016) & 4 & 1 & 1 & 6 & $(20)$ \\
Wu (2017) & 3 & 1 & 3 & 7 & $(21)$ \\
Liang (2018) & 2 & 1 & 3 & 6 & $(22)$ \\
Gao (2018) & 4 & 1 & 3 & 8 & $(23)$ \\
Fang (2019) & 4 & 2 & 1 & 7 & $(15)$ \\
Luo (2019) & 4 & 1 & 1 & 6 & $(17)$ \\
Hu (2019) & 3 & 2 & 3 & 8 & $(18)$ \\
\hline
\end{tabular}

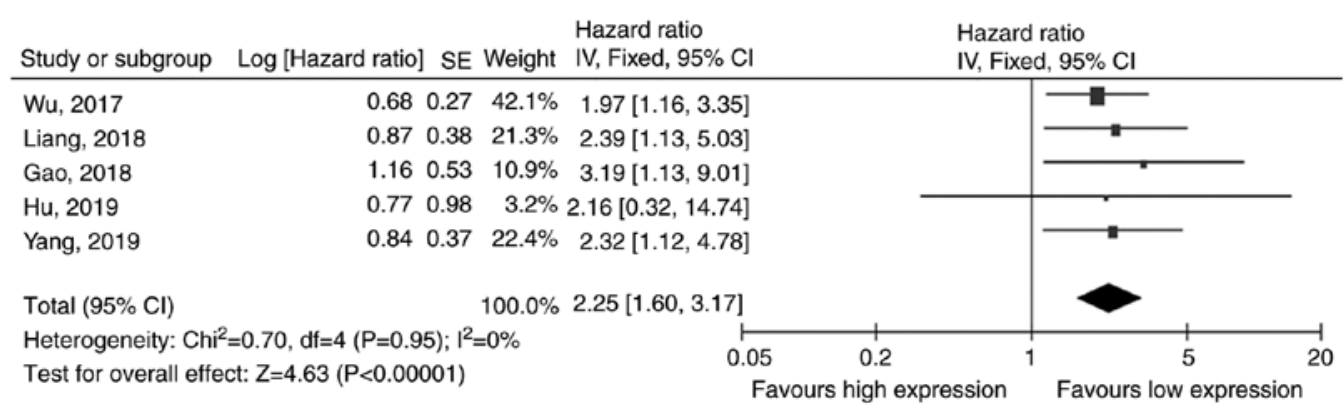

Figure 2. Forest plot for the association between cancer susceptibility candidate 9 expression and overall survival. IV, inverse variance; SE, standard error; df, degrees of freedom.

recent publication was from July 2019. The mean sample size of these studies was 121 (range, 20-128). A total of 3 studies were on ESCC (21-23), and one study each was on OSCC (12), GC (15), CRC (17), OC (18) and esophageal cancer (EC) (20). The expression levels of CASC 9 had been detected by reverse transcription-quantitative PCR $(15,17,18,20-23)$ and in situ hybridization (12). The internal controls differed across the studies and included 18S ribosomal RNA (20), $\beta$-actin (17), U6 (18) and GAPDH $(12,22,23)$. Based on the cut-off threshold, all patients with cancer were divided into the CASC9 high and CASC9 low groups in each included study. The characteristics of the studies are summarized in Table I and the functional roles or molecular mechanisms of CASC9 in Table II. The survival outcomes of cancer patients were assessed in terms of OS (12,18,21-23), DFS (22) and PFS (18) in 5 (62.5\%), 1 (12.5\%) and $1(12.5 \%)$ studies, respectively. The quality of all included studies was good, each with a NOS score $\geq 6$ (Table III).

Association between CASC 9 expression and OS. A total of 5 studies $(12,18,21-23)$ including 461 patients reported on the association between CASC 9 expression and OS rates. Since there was no obvious heterogeneity in these studies $\left(\mathrm{I}^{2}=0 \%\right)$, the fixed-effects model was applied. The pooled results revealed that cancer patients with high CASC9 expression had a shorter OS compared to those with low levels $(\mathrm{HR}=2.25$, 95\% CI: 1.60-3.17, P<0.001; Fig. 2).

Association between CASC9 expression and clinicopathological features. The association of CASC9 expression with sex (male vs. female), age ( $<60 \mathrm{vs.} \geq 60$ years), tumor size ( $\leq 4 \mathrm{vs} .>4 \mathrm{~cm}$ ), depth of invasion (T3+T4 vs. T1+T2), degree of tumor differentiation (poor vs. well/moderate), lymph node metastasis (presence vs. absence) and clinical stage (III+IV vs. I+II) were systematically evaluated. The depth of invasion $(\mathrm{OR}=2.66,95 \% \mathrm{CI}: 1.72-4.14, \mathrm{P}<0.001)$, tumor differentiation $(\mathrm{OR}=2.44,95 \% \mathrm{CI}: 1.24-4.78, \mathrm{P}=0.009)$, lymph node metastasis $(\mathrm{OR}=3.42,95 \% \mathrm{CI}: 1.98-5.92, \mathrm{P}<0.001)$ and clinical stage $(\mathrm{OR}=3.21,95 \% \mathrm{CI}: 2.21-4.66, \mathrm{P}<0.001)$ were significantly associated with high levels of CASC9, whereas sex, age and tumor size did not exhibit any significant association ( $\mathrm{P}>0.05$; Fig. 3).

Sensitivity analysis. The sensitivity analysis indicated an insignificant impact of omitting each study one by one on the overall results, which validated the credibility of the meta-analysis (Fig. 4). Due to the small number of studies reporting on DFS or PFS, sensitivity analysis was not performed for these variables.

Publication bias. Begg's funnel plot and Egger's tests revealed no publication bias for OS, sex, age, tumor size, depth of invasion, lymph node metastasis and clinical stage (Table IV). However, the asymmetrical funnel plot (Fig. 5A) and Egger's test $(\mathrm{P}=0.016$; Fig. $5 \mathrm{~B})$ suggested a publication bias in terms of tumor differentiation. Therefore, the 'trim and fill' method (29) was used to trace the possible influencing factors. As indicated in Fig. 5C, two studies were identified at the bottom of the adjusted funnel plot. The adjusted overall effect size $(\mathrm{OR}=6.208$, 95\% CI: 3.196-12.056, $\mathrm{P}<0.001)$ for tumor differentiation was not significantly different compared 


\section{A}

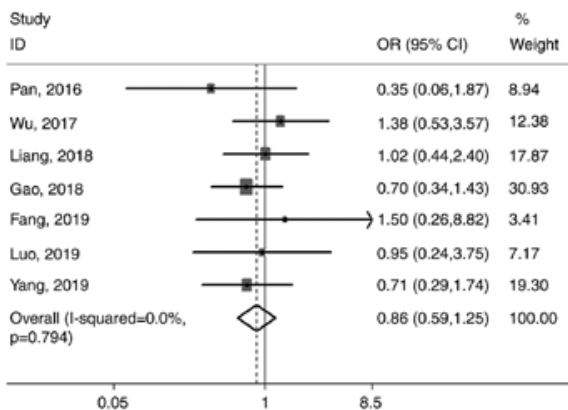

$\mathrm{C}$
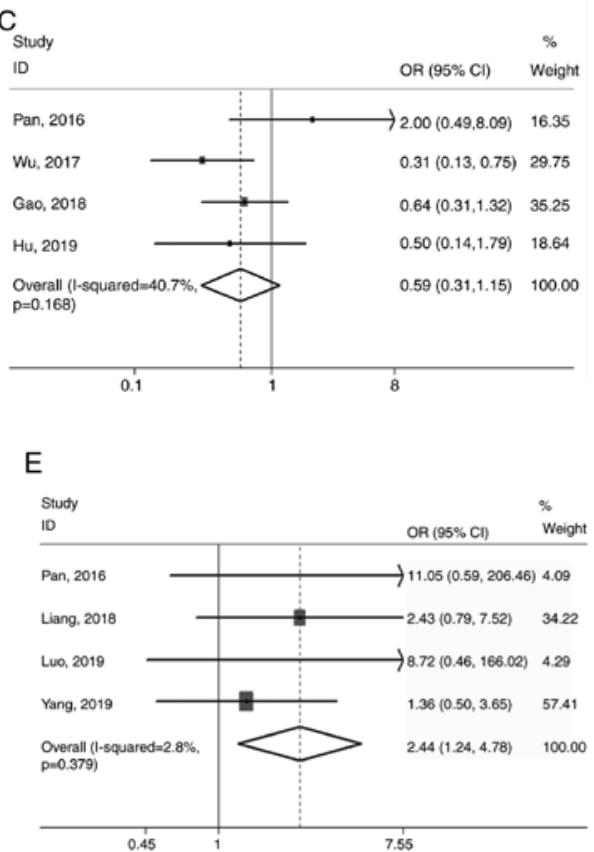

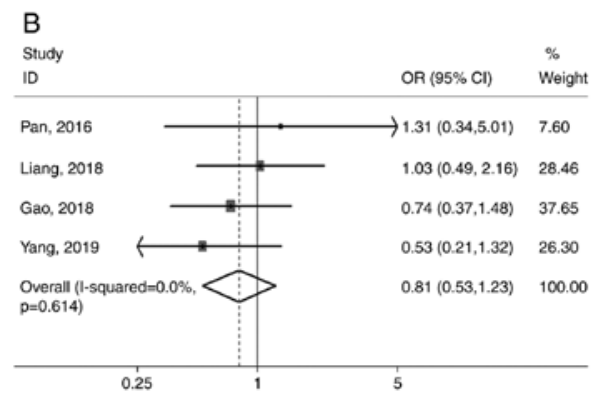

D
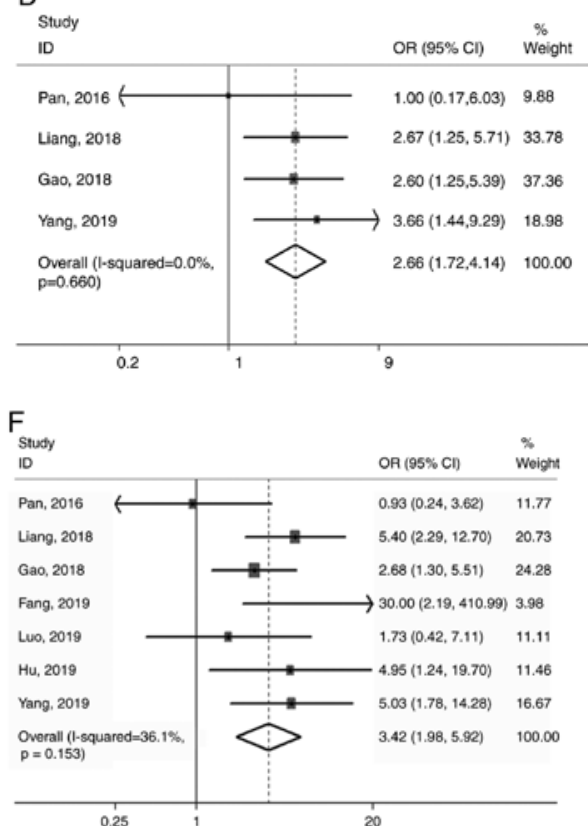

G

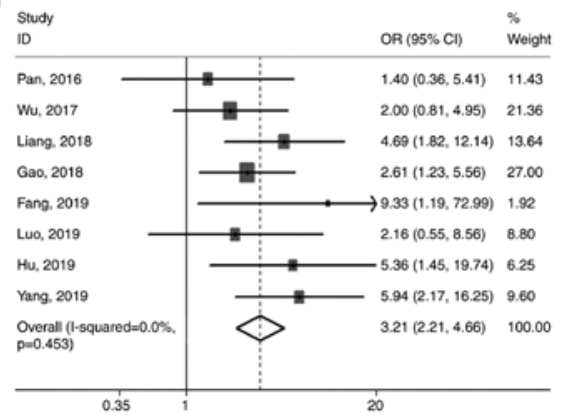

Figure 3. Forest plots for the association between cancer susceptibility candidate 9 expression and clinicopathological features, including (A) sex, (B) age, (C) tumor size, (D) depth of invasion, (E) tumor differentiation, (F) lymph node metastasis and (G) clinical stage. OR, odds ratio.

with that before adjustment (OR, 8.449; 95\% CI, 3.861-13.037; $\mathrm{P}=0.016$ ), and the funnel plot did not exhibit any evident asymmetry following correction, indicating robust results.

\section{Discussion}

Studies increasingly indicate the involvement of lncRNAs in biological processes, including the cell cycle, proliferation, apoptosis and tumorigenesis (30-32). There is also evidence confirming that IncRNAs are frequently dysregulated in human cancers and that these abnormally expressed lncRNAs are prognostically relevant (33-38). Therefore, identifying novel cancer-associated lncRNAs may provide predictors of the prognosis of cancer patients.

The IncRNA CASC9 is aberrantly expressed in various malignancies and associated with cancer development and progression, although its exact functional role depends on the cancer type. For instance, CASC9 is an established oncogene in ESCC cells, wherein it downregulates the programmed cell death 4, upregulates recombinant laminin $\gamma 2$ and promotes epithelial-mesenchymal transition (21-23). Furthermore, CASC9 knockdown inhibited the proliferation, cell cycle, migration and invasion of ESCC cells. Silencing of CASC9 in GC cells facilitated the degradation of $\mathrm{B}$ lymphoma Moloney murine leukemia virus 
A

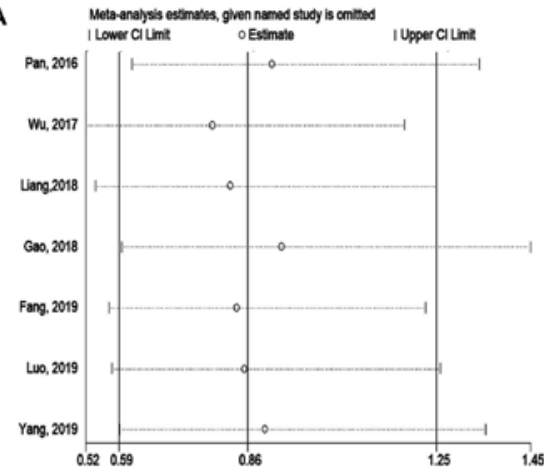

C

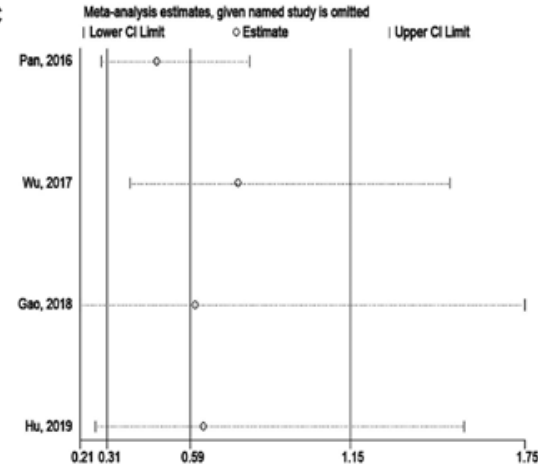

E Meta-analysis estimates, given named study is omitted Pan, 2016 ILower $\mathrm{Cl}$ limit $\circ$ Estimate IUpper $\mathrm{Cl}$ limit
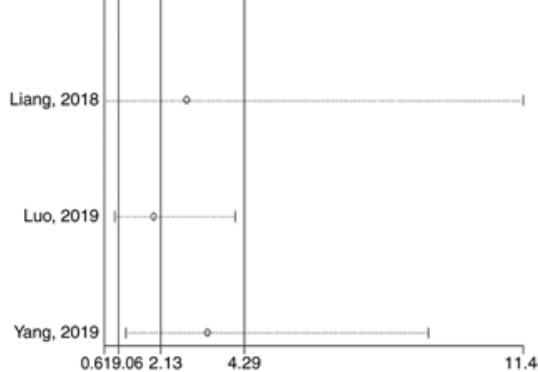

G Meta-analysis estimates, given named study is omitted

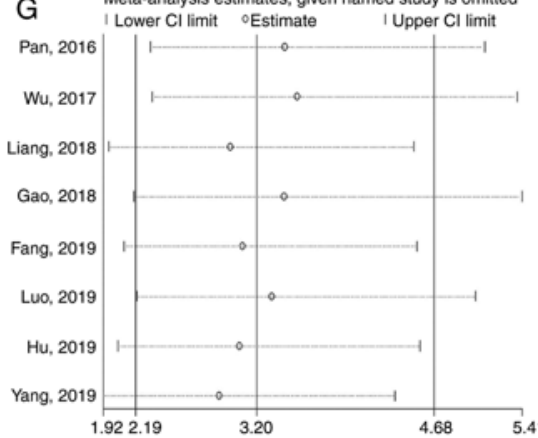

B

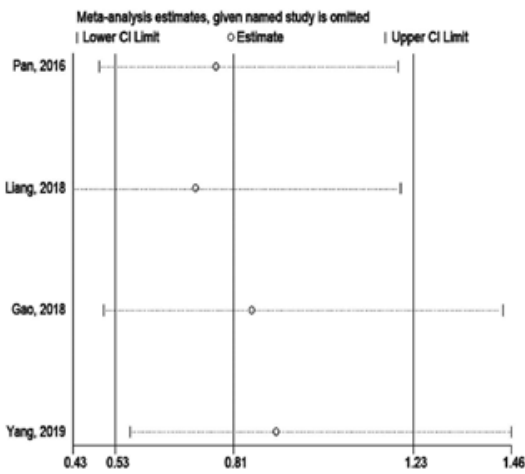

D

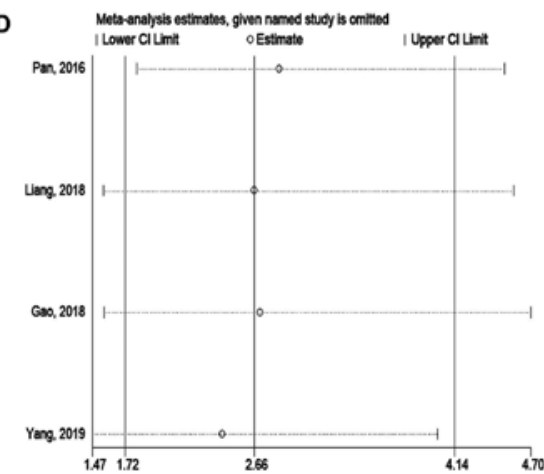

M Meta-analysis estimates, given named study is omitted
ILower $\mathrm{Cl}$ limit
○Estimate IUpper Cl limit

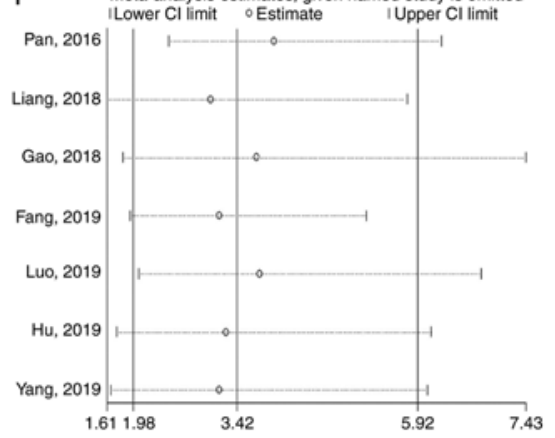

H I Loweranalysis estimates, given named study is omitted

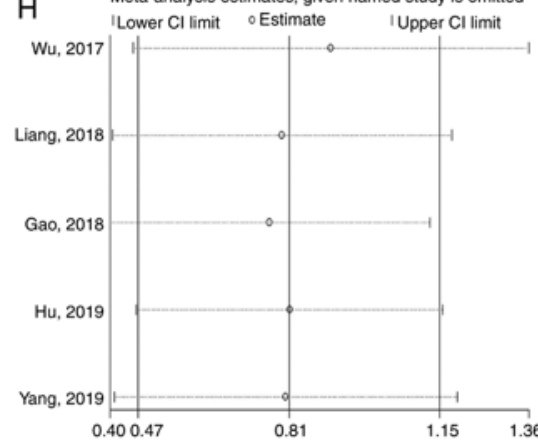

Figure 4. Sensitivity analysis for all of the outcomes. (A) Sex, (B) age, (C) tumor size, (D) depth of invasion, (E) tumor differentiation, (F) lymph node metastasis, $(\mathrm{G})$ clinical stage and $(\mathrm{H})$ overall survival.

insertion region 1, leading to apoptosis (15). Furthermore, CASC9 promoted the proliferation of CRC cells and inhibited apoptosis by interacting with cleavage and polyadenylation specificity factor subunit 3 and activating transforming growth factor- $\beta 2$ signaling (17). Hu et al (18) indicated that CASC9 increased the proliferation, invasion and migration of $\mathrm{OC}$ cells by upregulating lin-7 homolog A via microRNA-758-3p. Jiang et al (39) revealed that CASC9 could promote the growth, metastasis and chemoresistance of $\mathrm{BC}$ cells via binding to enhancer of zeste homolog 2 and mediating the expression of multidrug resistance protein 1. Yang et al (12) demonstrated that high expression of CASC9 in OSCC cells enhanced proliferation and inhibited apoptosis through the AKT/mTOR pathway. The results of the present meta-analysis also suggested an oncogenic role of CASC9, 
Table IV. Publication bias of all outcomes assessed by Begg's test and Egger's test.

\begin{tabular}{|c|c|c|c|c|c|}
\hline Outcome & $\begin{array}{c}\text { Number of } \\
\text { studies/patients }\end{array}$ & Estimates & $\begin{array}{l}\text { Begg's test } \\
\text { (P-value) }\end{array}$ & $\begin{array}{l}\text { Egger's test } \\
\text { (P-value) }\end{array}$ & $\begin{array}{l}\text { Publication } \\
\text { bias }\end{array}$ \\
\hline Sex & $7 / 522$ & $\mathrm{OR}+95 \% \mathrm{CI}$ & 0.548 & 0.698 & Not significant \\
\hline Age & $4 / 371$ & $\mathrm{OR}+95 \% \mathrm{CI}$ & 0.734 & 0.620 & Not significant \\
\hline Tumor size & $4 / 306$ & $\mathrm{OR}+95 \% \mathrm{CI}$ & 0.734 & 0.402 & Not significant \\
\hline Depth of invasion & $4 / 371$ & $\mathrm{OR}+95 \% \mathrm{CI}$ & 1.000 & 0.522 & Not significant \\
\hline Tumor differentiation & $4 / 283$ & $\mathrm{OR}+95 \% \mathrm{CI}$ & 0.308 & 0.016 & Significant \\
\hline Lymph node metastasis & $7 / 474$ & $\mathrm{OR}+95 \% \mathrm{CI}$ & 0.548 & 0.152 & Not significant \\
\hline Clinical stage & $8 / 565$ & $\mathrm{OR}+95 \% \mathrm{CI}$ & 0.266 & 0.298 & Not significant \\
\hline OS & $5 / 461$ & $\mathrm{HR}+95 \% \mathrm{CI}$ & 0.462 & 0.350 & Not significant \\
\hline
\end{tabular}

HR, hazard ratio; OR, odds ratio; OS, overall survival.
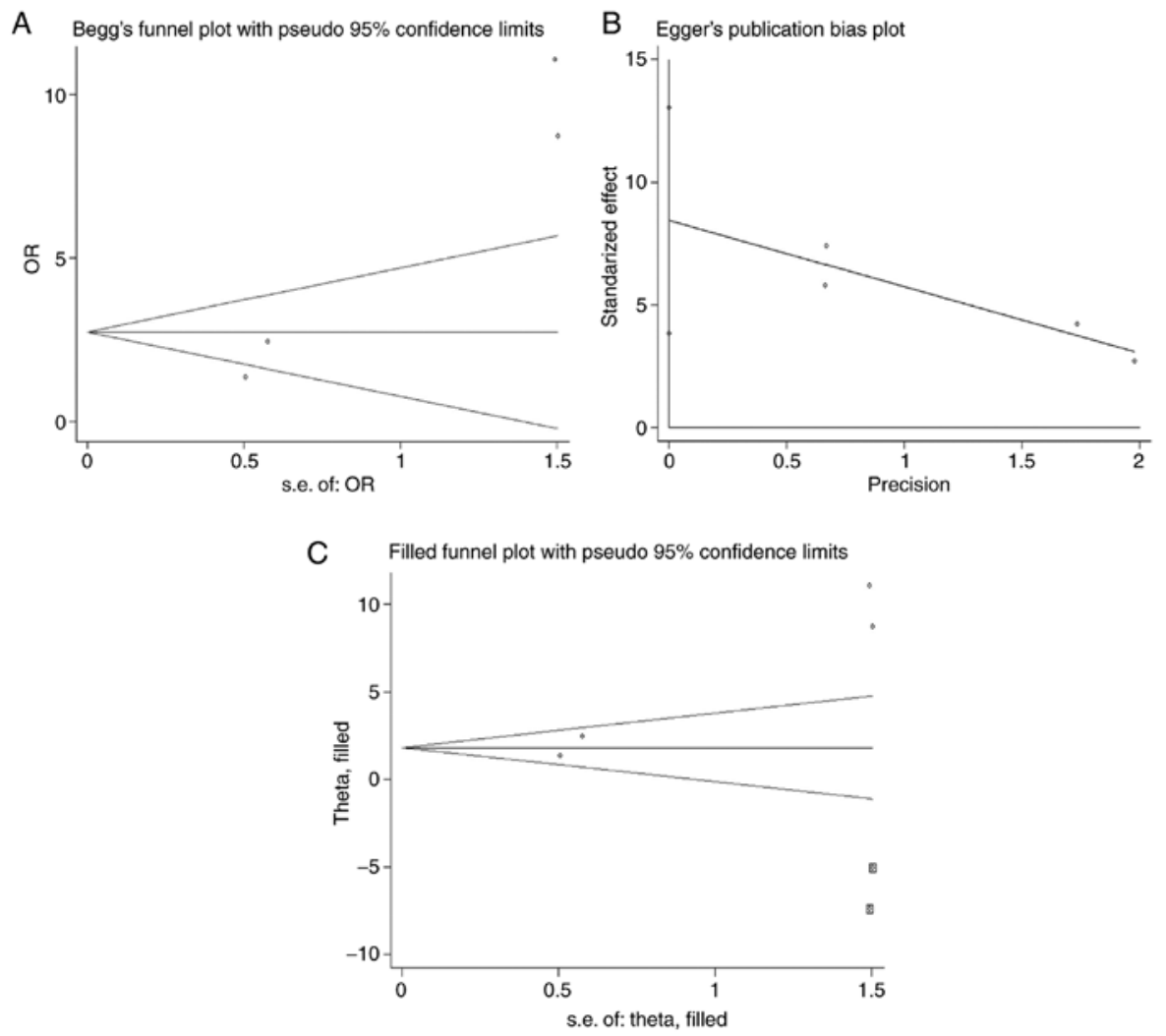

Figure 5. Funnel plot indicating publication bias for tumor differentiation. (A) Begg's funnel plot. (B) Egger's publication bias plot. (C) Begg's funnel plot adjusted using the 'trim and fill' method (indicates observed studies; indicates imputed studies).

since its high expression levels in solid tumors were associated with pro-oncogenic effects, including like proliferation $(12,17,18,21,23)$, invasion $(18,20,22,23)$, migration $(18,20,22,23)$ and suppression of apoptosis $(12,15,17)$, via different targets or signaling pathways depending on the cancer type.

However, the prognostic significance of CASC9 in human cancers remains largely inconclusive. According to the present meta-analysis, increased expression of CASC9 portends shorter OS, deeper tumor invasion, poor tumor differentiation, lymph node metastasis and advanced clinical stage. Since only one study (23) analyzed the impact of increased CASC9 expression on the DFS of ESCC patients and one study (18) reported the influence of high CASC9 expression on the PFS of patients with OC, it was not possible to determine a definite association between CASC9 expression and DFS/PFS. In addition, a publication bias in terms of tumor differentiation was observed. Nevertheless, the 'trim and fill' method indicated robust results, and the sensitivity analysis additionally demonstrated the consistency and reliability of the results.

To the best of our knowledge, the present study was the first meta-analysis to evaluate the prognostic and clinicopathological significance of CASC9 in multiple cancer types. The results are highly relevant from a clinical point of view and suggest that 
CASC9 may be a promising therapeutic target for multiple cancer types. However, several limitations of the present study should be taken into consideration. First, since all populations in the included studies were Chinese, the results should be extrapolated with caution to populations in other regions. In addition, only articles published in the English language were included, and relevant studies in other languages may have been omitted. Furthermore, the number of included studies and cancer types in the present meta-analysis was relatively small, which may have affected the estimated predictive ability of CASC9. Likewise, the HRs and 95\% CIs from three studies were indirectly extracted by extrapolating the Kaplan-Meier survival curves, which may have reduced the accuracy of the results. Finally, there was no consensus on the cut-off for CASC9 overexpression in tumor tissues across the different studies, which may have led to potential heterogeneity among these studies.

To conclude, CASC9 overexpression is predictive of poor $\mathrm{OS}$ in cancer patients and is significantly associated with deeper tumor invasion, poor tumor differentiation, lymph node metastasis and advanced clinical stage. It is a promising prognostic biomarker for cancer and requires to be validated by multi-center studies with large patient cohorts.

\section{Acknowledgements}

Not applicable.

\section{Funding}

No funding was received.

\section{Availability of data and materials}

The datasets used and/or analyzed during the present study are available from the corresponding author on reasonable request.

\section{Authors' contributions}

HYD and HSL conceived and designed the current study. HYD and XD conducted data collection and extraction. HYD analyzed the data. HYD and HSL drafted the manuscript. All authors read and approved the final manuscript.

\section{Ethics approval and consent to participate}

Not applicable.

\section{Patient consent for publication}

Not applicable.

\section{Competing interests}

The authors declare that they have no competing interests.

\section{References}

1. Bray F, Ferlay J, Soerjomataram I, Siegel RL, Torre LA and Jemal A: Global cancer statistics 2018: GLOBOCAN estimates of incidence and mortality worldwide for 36 cancers in 185 countries. CA Cancer J Clin 68: 394-424, 2018.
2. Ulitsky I and Bartel DP: lincRNAs: genomics, evolution, and mechanism. Cell 154: 26-46, 2013.

3. Chen LL: Linking long noncoding RNA localization and function. Trends Biochem Sci 41: 761-772, 2016.

4. Hung T and Chang HY: Long noncoding RNA in genome regulation: Prospects and mechanisms. RNA Biol 7: 582-585, 2010.

5. Yamashita A, Shichino Y and Yamamoto M: The long non-coding RNA world in yeasts. Biochim Biophys Acta 1859: 147-154, 2016.

6. Loewen G, Jayawickramarajah J, Zhuo Y and Shan B: Functions of IncRNA HOTAIR in lung cancer. J Hematol Oncol 7: 90, 2014.

7. Iguchi T, Uchi R, Nambara S, Saito T, Komatsu H, Hirata H, Ueda M, Sakimura S, Takano Y, Kurashige J, et al: A long noncoding RNA, lncRNA-ATB, is involved in the progression and prognosis of colorectal cancer. Anticancer Res 35: 1385-1388, 2015.

8. Flicek P, Ahmed I, Amode MR, Barrell D, Beal K, Brent S, Carvalho-Silva D, Clapham P, Coates G, Fairley S, et al: Ensembl 2013. Nucleic Acids Res 41 (D1): D48-D55, 2013.

9. Klingenberg M, Groß M, Goyal A, Polycarpou-Schwarz M, MierschT,Ernst AS,LeupoldJ,PatilN, WarnkenU,AllgayerH, etal: The long noncoding RNA cancer susceptibility 9 and RNA binding protein heterogeneous nuclear ribonucleoprotein $\mathrm{L}$ form a complex and coregulate genes linked to AKT signaling. Hepatology 68: 1817-1832, 2018.

10. Cao W, Wu W, Shi F, Chen X, Wu L, Yang K, Tian F, Zhu M, Chen $\mathrm{G}$, Wang W, et al: Integrated analysis of long noncoding RNA and coding RNA expression in esophageal squamous cell carcinoma. Int J Genomics 2013: 480534-480543, 2013.

11. Su X, Li G and Liu W: The long noncoding RNA cancer susceptibility candidate 9 promotes nasopharyngeal carcinogenesis via stabilizing HIF1a. DNA Cell Biol 36: 394-400, 2017.

12. Yang Y, Chen D, Liu H and Yang K: Increased expression of lncRNA CASC9 promotes tumor progression by suppressing autophagy-mediated cell apoptosis via the AKT/mTOR pathway in oral squamous cell carcinoma. Cell Death Dis 10: 41-56, 2019.

13. Gao L, Guo YN, Zeng JH, Ma FC, Luo J, Zhu HW, Xia S, Wei KL and Chen G: The expression, significance and function of cancer susceptibility candidate 9 in lung squamous cell carcinoma: A bioinformatics and in vitro investigation. Int J Oncol 54: 1651-1664, 2019.

14. Shao G, Wang M, Fan X, Zhong L, Wang Z, Zhang P and Ji S: lncRNA CASC9 positively regulates $\mathrm{CHK} 1$ to promote breast cancer cell proliferation and survival through sponging the miR 195/497 cluster. Int J Oncol 54: 1665-1675, 2019.

15. Fang J, Chen W and Meng XL: IncRNA CASC9 Suppressed the Apoptosis of gastric cancer cells through regulating BMI1. Pathol Oncol Res 10: 22-29, 2019.

16. Yu X, Lin Y, Sui W, Zou Y and Lv Z: Analysis of distinct long noncoding RNA transcriptional fingerprints in pancreatic ductal adenocarcinoma. Cancer Med 6: 673-680, 2017.

17. Luo K, Geng J, Zhang Q, Xu Y, Zhou X, Huang Z, Shi KQ, Pan C and $\mathrm{Wu} \mathrm{J}$ : IncRNA CASC9 interacts with CPSF3 to regulate TGF- $\beta$ signaling in colorectal cancer. J Exp Clin Cancer Res 38: 249-264, 2019.

18. Hu X, Li Y, Kong D, Hu L, Liu D and Wu J: Long noncoding RNA CASC9 promotes LIN7A expression via miR-758-3p to facilitate the malignancy of ovarian cancer. J Cell Physiol 234: 10800-10808, 2019.

19. Zhang J, Wang Q and Quan Z: Long non-coding RNA CASC9 enhances breast cancer progression by promoting metastasis through the meditation of miR-215/TWIST2 signaling associated with TGF- $\beta$ expression. Biochem Biophys Res Commun 515: 644-650, 2019.

20. Pan Z, Mao W, Bao Y, Zhang M, Su X and Xu X: The long noncoding RNA CASC9 regulates migration and invasion in esophageal cancer. Cancer Med 5: 2442-2447, 2016.

21. Wu Y, Hu L, Liang Y, Li J, Wang K, Chen X, Meng H, Guan X, Yang K and Bai Y: Up-regulation of IncRNA CASC9 promotes esophageal squamous cell carcinoma growth by negatively regulating PDCD4 expression through EZH2. Mol Cancer 16: 150-163, 2017.

22. Liang Y, Chen X, Wu Y, Li J, Zhang S, Wang K, Guan X, Yang K and Bai Y: IncRNA CASC9 promotes esophageal squamous cell carcinoma metastasis through upregulating LAMC2 expression by interacting with the CREB-binding protein. Cell Death Differ 25: 1980-1995, 2018.

23. Gao GD, Liu XY, Lin Y, Liu HF and Zhang GJ: 1ncRNA CASC9 promotes tumorigenesis by affecting EMT and predicts poor prognosis in esophageal squamous cell cancer. Eur Rev Med Pharmacol Sci 22: 422-429, 2018. 
24. Moher D, Liberati A, Tetzlaff J, Altman DG and Group P; PRISMA Group: Preferred reporting items for systematic reviews and meta-analyses: The PRISMA statement. PLoS Med 6: e1000097, 2009.

25. Tierney JF, Stewart LA, Ghersi D, Burdett S and Sydes MR: Practical methods for incorporating summary time-to-event data into meta-analysis. Trials 8: 16, 2007.

26. Stang A: Critical evaluation of the Newcastle-Ottawa scale for the assessment of the quality of nonrandomized studies in meta-analyses. Eur J Epidemiol 25: 603-605, 2010.

27. Wong WC, Cheung CS and Hart GJ: Development of a quality assessment tool for systematic reviews of observational studies (QATSO) of HIV prevalence in men having sex with men and associated risk behaviours. Emerg Themes Epidemiol 5: 23-26, 2008.

28. Begg CB and Mazumdar M: Operating characteristics of a rank correlation test for publication bias. Biometrics 50: 1088-1101, 1994.

29. Duval S and Tweedie R: Trim and fill: A simple funnel-plot-based method of testing and adjusting for publication bias in meta-analysis. Biometrics 56: 455-463, 2000

30. Mathieu EL, Belhocine M, Dao LT, Puthier D and Spicuglia S: Functions of lncRNA in development and diseases. Med Sci (Paris) 30: 790-796, 2014 (In French).

31. Peng WX, Koirala P and Mo YY: lncRNA-mediated regulation of cell signaling in cancer. Oncogene 36: 5661-5667, 2017.

32. Gao P and Wei GH: Genomic insight into the role of lncRNA in cancer susceptibility. Int J Mol Sci 18: 1239-1249, 2017.

33. Yarmishyn AA and Kurochkin IV: Long noncoding RNAs: A potential novel class of cancer biomarkers. Front Genet 6: $145-154,2015$
34. Evans JR, Feng FY and Chinnaiyan AM: The bright side of dark matter: lncRNAs in cancer. J Clin Invest 126: 2775-2782, 2016.

35. Zhao QS, Li L, Zhang L, Meng XW, Li LL, Ge XF and Li ZP. Over-expression of 1ncRNA SBF2-AS1 is associated with advanced tumor progression and poor prognosis in patients with non-small cell lung cancer. Eur Rev Med Pharmacol Sci 20: 3031-3034, 2016.

36. Qi P and Du X: The long non-coding RNAs, a new cancer diagnostic and therapeutic gold mine. Mod Pathol 26: 155-165, 2013.

37. Wang JJ, Huang YQ, Song W, Li YF, Wang H, Wang WJ and Huang M: Comprehensive analysis of the lncRNA associated competing endogenous RNA network in breast cancer. Oncol Rep 42: 2572-2582, 2019.

38. Wang X, Zhou X, Liu J, Liu Z, Zhang L, Gong Y, Huang J, Yu L, Wang Q, Yang C, et al: Genome wide investigation of the clinical implications and molecular mechanism of long noncoding RNA LINC00668 and protein coding genes in hepatocellular carcinoma. Int J Oncol 55: 860-878, 2019.

39. Jiang B, Li Y, Qu X, Zhu H, Tan Y, Fan Q, Jiang Y, Liao M and Wu X: Long noncoding RNA cancer susceptibility candidate 9 promotes doxorubicin resistant breast cancer by binding to enhancer of zeste homolog 2. Int J Mol Med 42: 2801-2810, 2018.

(i) () () This work is licensed under a Creative Commons

Attribution-NonCommercial-NoDerivatives 4.0 International (CC BY-NC-ND 4.0) License. 